\title{
Heat Transfer Coefficient Prediction of a Porous Material by Implementing a Machine Learning Model on a CFD Data Set
}

\author{
Edgar Avalos-Gauna, León Palafox-Novack \\ Universidad Panamericana, Campus México \\ Augusto Rodin 498, 03920, Ciudad de México, México \\ eavalos@up.edu.mx; lpalafox@up.edu.mx
}

\begin{abstract}
During many years, the search for new and improved materials has been an arduous task. It has mainly focused on experimentation and in recent years on computer aided techniques (i.e. numerical simulation). These two approaches defined the way material science works. Yet, both techniques have shown cost-efficiency disadvantages. Optimization algorithms, like the ones used in machine learning, have proven to be an alternative tool when dealing with lots of data and finding a particular solution. Even though the use of machine learning is a well stablished technique in other fields, its application in material science is relatively new. Material Informatics provides a new approach to analyse materials such as porous metals by employing previous data sets. This paper studies a new technique to predict the heat transfer coefficient of an open-cell porous structure while running water passes through the material. A CFD data set was employed by a Machine Learning technique in order to establish a relationship between the input parameters (porosity, pore size, pore distribution and flow rate) and the heat transfer coefficient of the sample. The results obtained from the analyses were compared with previous findings, concluding that by utilising a Machine Leaning technique is possible to obtain a more accurate and much better fit model.
\end{abstract}

Keywords: Heat transfer coefficient, Porosity, Machine Learning, Materials informatics.

\section{Introduction}

Study of materials science and materials design have been linked to previous experiences and trial-error analyses since the beginning [1]. From that, empirical correlations had been obtained between processing conditions and desired performances [2]. Therefore, the main objective of using this approach is to obtain and enhancement of the material by modifying its known properties. This typical materials selection approach is widely accepted to address product-level performance requirements. In such a case, new or improved multifunctional materials enrich many industries. These materials may have good properties, however, it does not necessarily mean that they have the right balance of properties needed for specific engineering applications [1]. For instance, in high performance alloys and composite materials, maximum performance is often achieved within a specific range of properties with a small variance [3].

More recently, computational techniques are included in evaluating the necessary performance metrics to support materials design [4]. This approach is known as numerical simulation (e.g. finite element models) and it has been used during the past few decades in order to analyse different material properties. Yet, the main issue in obtaining relevant properties from a numerical model, is the correct selection of the main microstructure measures that could have a higher influence on them. Nowadays, this selection is made based on experience [5]. In addition, the time and financial investment required in any of these two approaches, often limits the development of new materials [6]. Therefore, new R\&D techniques must be developed in order to provide a cost-effective solution.

Machine Learning (ML) therefore, can help us to determine desired material properties or design directly predicted based on first principle calculations. By employing different learning algorithms, these data sets are going to be used as training sets so every combination is considered [7]. The learning algorithms can be classified in 3 subcategories based on their learning styles [8]:

- Supervised learning (Decision Tree, Artificial Neural Network, Support Vector Machines)

- Unsupervised learning (Clustering, Associate Rules, etc.)

- Semi-supervised learning techniques (Generative models, Low-density separation, etc.) 
As a general rule, the efficiency of the algorithm can be measured on how well it performs while optimizing the problem on which it is tested [9].

ML techniques and density functional theory come together in order to constitute a new field of research: Materials informatics. Given the different algorithms in existence, materials informatics allows to study complex and large datasets in a statistically complete, and yet tangibly and meaningful manner [6]. Thus, Materials informatics is one of the most promising techniques that is more cost-efficient [10], and can be used on large material databases for material predictions [7]. The increased usage of materials informatics has been occurring during the past decade [6]. This has led to huge efforts made by the materials science community in order to create open data bases to accelerate materials development and facilitates the discovery of new materials [11]. For example, the Materials Genome Initiative (MGI) was proposed to reduce the cost and development time of materials discovery, optimization, and deployment [12].

In the past few years, some studies have started to utilise large data sets associated to certain material properties. These data sets have been used as training sets for different ML techniques in order to predict targeted properties on sets of unknown data [13]. Conduit et al. [14] developed a neural network in order to model, discover, and optimize different types of new alloys while considering eleven different features. Their trained model was tested with experimental data and the predicted nickel-base polycrystalline alloy offered an ideal performance with the target properties. Properties such as yield stress and oxidation resistance were experimentally verified. Artrith \& Urban [15] implemented the Behler-Parrinello approach which is based on artificial neural networks with the free and open-source atomic energy network (ænet) package. The modelling and training of the algorithm was presented and predicted a promising performance for a crystal phase $\mathrm{TiO}_{2}$.

The wide variety of pore parameters that can be obtained during rigorous quantification provides large amounts of data that need to be analysed. All these pore related properties are fundamentally important due to the fact that they generate the permeability capability of an open-cell structure. This permeability allowsfluids to flow through its internal network of the cells [16]. As the porous copper is being heated, this open structure allows the material to transfer heat to the running fluid. Conduction and forced convection are the two main ways of energy transfer on this system. Radiation can be neglected as it has been reported on the literature that heat transfer on porous metals only occurs at higher temperatures above 500K [17]. Therefore the importance of calculate the heat transfer coefficient of the samples efficiently.

In this investigation, a ML approach is used to study the pore-scale features of a given porous metal. In order to have an optimum selection of the porous material in order to calculate the heat transfer coefficient, information related to the structure (i.e. pore sizes, porosity, metal particle size) and the flow rate of the running fluid are considered.

\section{Methodology}

The main purpose of this paper is to demonstrate that data science tools are ideally suited to tackle the permeability calculation problem outlined above. One of the key aspects of materials informatics is that it lowers the costs of $R \& D$, by not having to use more experimental tests, and by reducing the computational usage.

Fig. 1 illustrates the flow chart of the ML process, which entails 4 different stages: pre-processing, learning, evaluation and prediction. The ML stages are explained as follows:

Pre-processing, consisting of data preparation and descriptor selector. For the former one, generating a dataset that comprises of the features (independent variables) and the targets or descriptors (dependant variables) [12]. Descriptor or target selection consists of finding the most influential features by implementing different data extraction techniques and dimensionality reduction methods such as Principal Component Analysis (PCA) [6].

Learning phase, which includes model selection and evaluation. Extracting and validating the model, has to bear in mind that it has to fit the data set, without over-fitting or under fitting it. Various regression methods are implemented and by cross validation the most optimal is selected[5].

Evaluation, and Prediction. As stated before, the most relevant features are obtained which then are used to make predictions on already known values. Once the predictions are sufficiently accurate, the model is used to predict targeted properties. This will derive in the development of specific type of materials. For materials informatics, the literature designates five descriptor categories: constitutional, topological, physicochemical, structural, and quantum-chemical [18]. For this paper, only topological properties related to the manufacturing process of the material were used. 


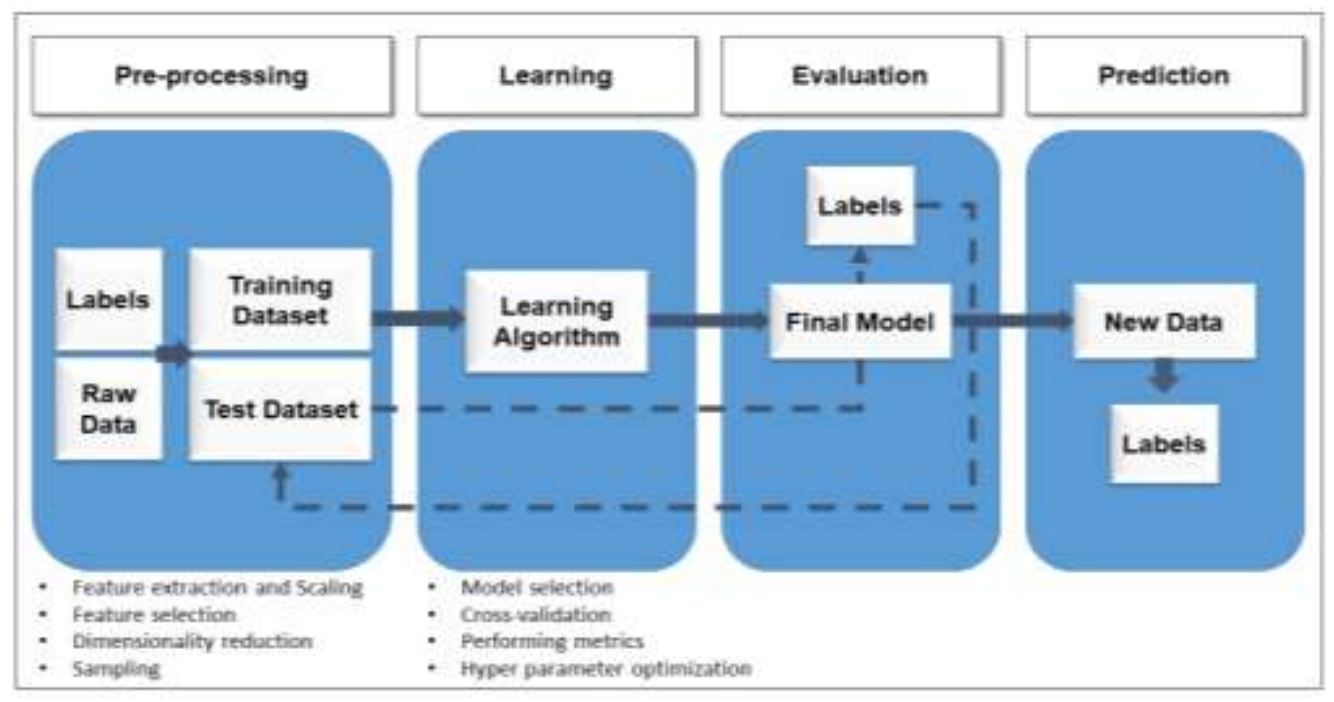

Fig. 1: Flow chart of the ML process.

Ohno [18] proposed a method to deal with the problem of having uneven dimensionality among the input vectors which often come in different size. This is also considered during the present study, as the dataset used do not include full data for the larger pore size $(1500 \mu \mathrm{m})$. The dataset consisted of 810 CFD simulations of fluid flow and heat transfer through forced convection on a representative elementary volume (REV) that characterised a porous metal [16]. The principal descriptors considered for this analysis were pore size (given by the diameter of the $\mathrm{K}_{2} \mathrm{CO}_{3}$ molecule, ranging from $400 \mu \mathrm{m}$ to $1500 \mu \mathrm{m}$ ), metal particle size $(\mathrm{Cu}$ diameter ranging from $20 \mu \mathrm{m}$ to $70 \mu \mathrm{m}$ ), porosity (ranging from $50 \%$ to $80 \%$ ) and flow rate (ranging from $0.2 \mathrm{l} / \mathrm{min}$ to $1.8 \mathrm{l} / \mathrm{min}$ ). These parameters are the most commonly used for a permeability analysis on a porous material. Coordination number is also used as possible descriptor. From the numerical simulations, the resulting values are total pressure and the heated plate temperature [19]. Finally, the dataset provided more information related to the target properties that were obtained during the post-processing analysis of the numerical data. Pressure drop, permeability, form drag coefficient, and heat transfer coefficient were obtained using Eq. (1) [20] and Eq. (2) [21]:

$$
\begin{gathered}
\frac{\Delta P}{L}=\frac{\mu}{K} u \\
h=\frac{J}{T_{p}-T_{w}}
\end{gathered}
$$

where $\Delta P$ is the total pressure, $L$ is the length of the REV, $\mu$ is the viscosity of water, $K$ is permeability, $u$ is the Darcian velocity, which can be obtained by dividing the flow rate by the cross sectional area of the REV, $h$ is the heat transfer coefficient, $J$ is the heat flux, $T_{p}$ is the temperature of the plate, and $T_{w}$ is the temperature of the water. For this analysis only the heat transfer coefficient is analysed. A correlation matrix was used in order to establish the relationship amongst all the parameters found in the dataset as shown in Fig. 2. 


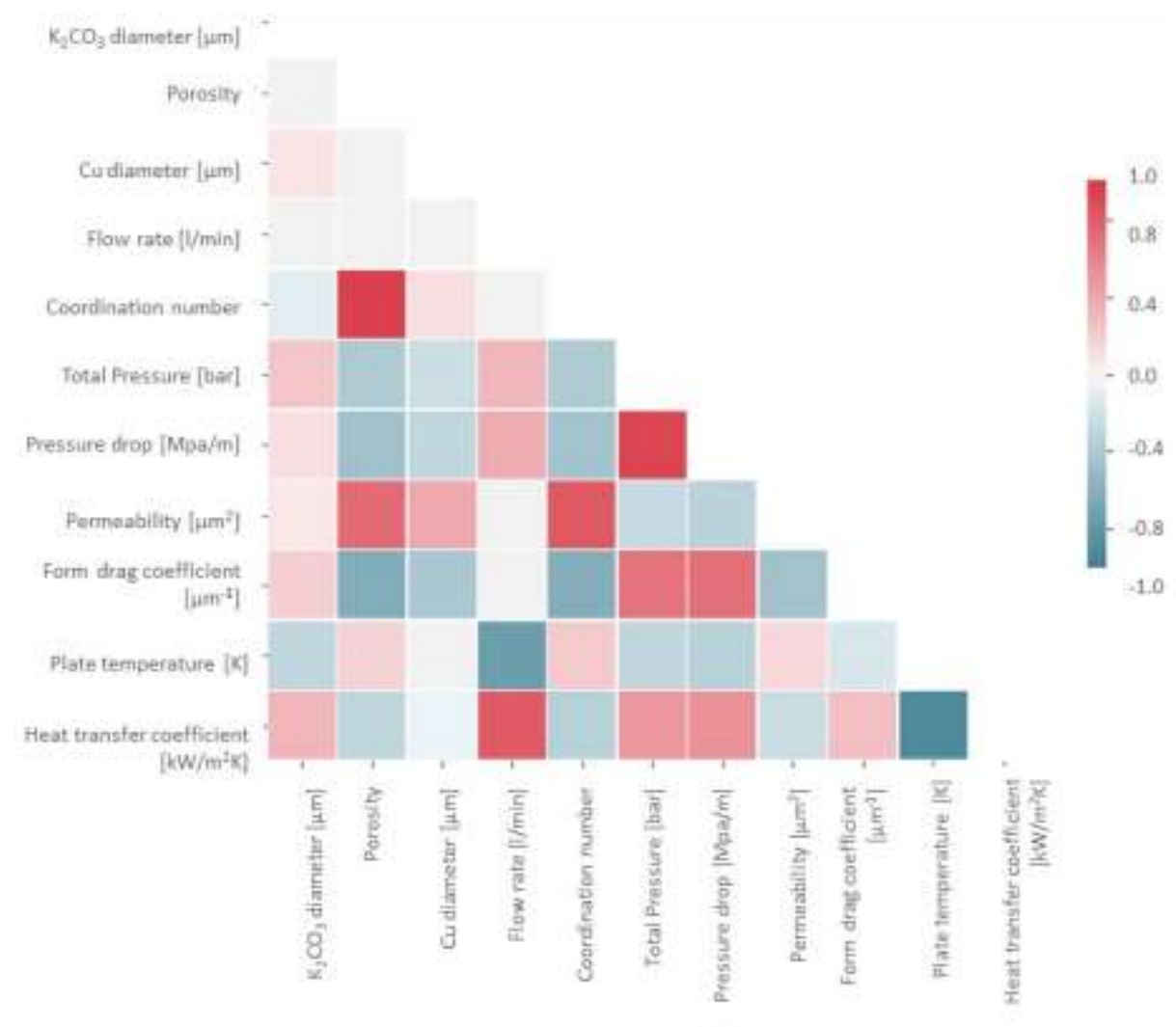

Fig. 2: Graphical representation of the relationship that exists amongst the parameters found on the dataset.

A positive relationship is marked in red whereas a negative is marked in blue. From Fig. 2 it is possible to establish the independent variables to be selected for the analysis: Porosity and Flow rate exhibited high influence on the other parameters. Some other parameters did not showed a strong relationship with other features of the geometrical model. Yet, pore size $\left(\mathrm{K}_{2} \mathrm{CO}_{3}\right.$ diameter) and metal particle size (Cu diameter) are also considered for the current analysis. The influence of these two elements on the heat transfer coefficient is in accordance to previous findings in the literature [22]. Other parameters such as coordination number or permeability come as a result of other parameters such as porosity or pore size. The former mentioned parameters (i.e. pore size and meta particle size) cannot be chosen at the beginning of the analysis like the later ones (i.e. coordination number and permeability). Therefore, the rest of the parameters are not considered for the model presented in this study.

\section{Results and discussion}

The numerical results for heat transfer coefficient from the dataset [16] were analysed against structural parameters reviewed in the CFD model. Porosity, pore size, metal particle size and flow rate were plotted against the heat transfer coefficient. The resulting graphs can be observed in Fig. 3. Porosity increases by moving to the next plot on the right while pore size increases by moving to the next plot downwards. The non-linear increasing trend for the 30 plots is related to the increasing flow rate. 

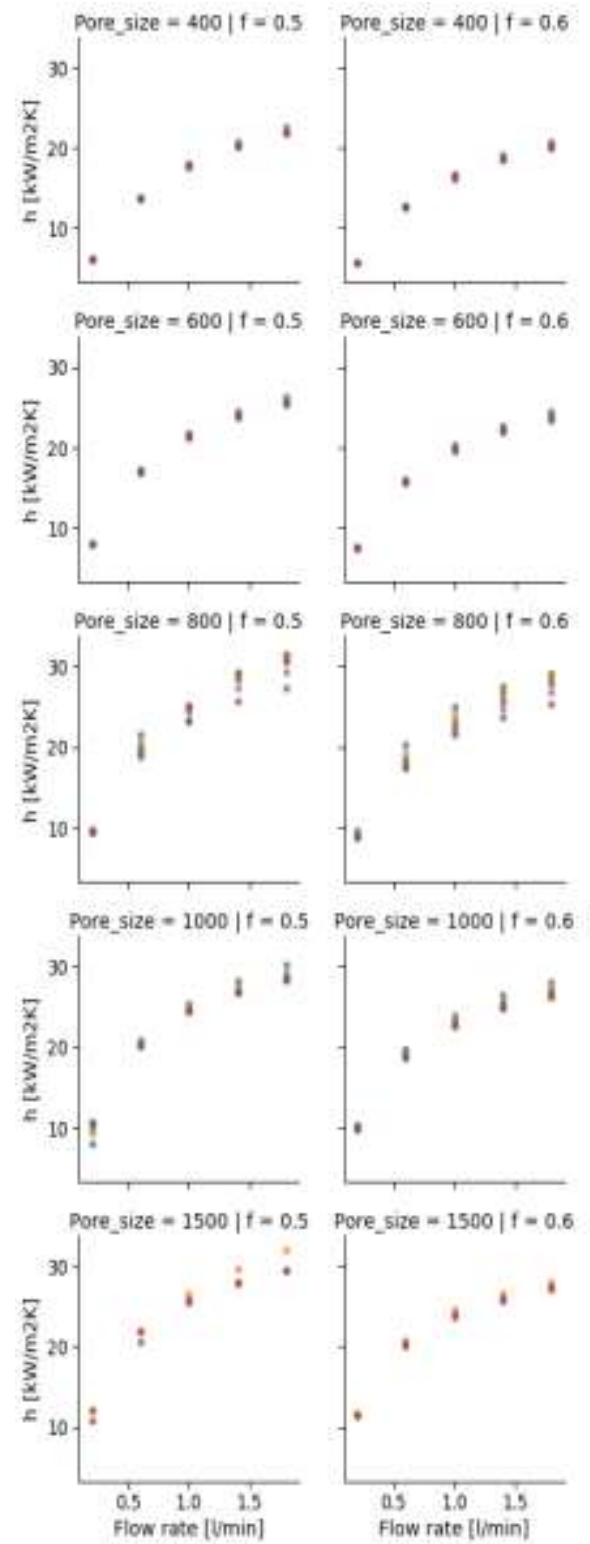

Pore size $=600 \mid f=0.6$

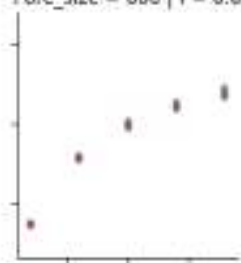

Pore size $=800 \mid t=0.6$
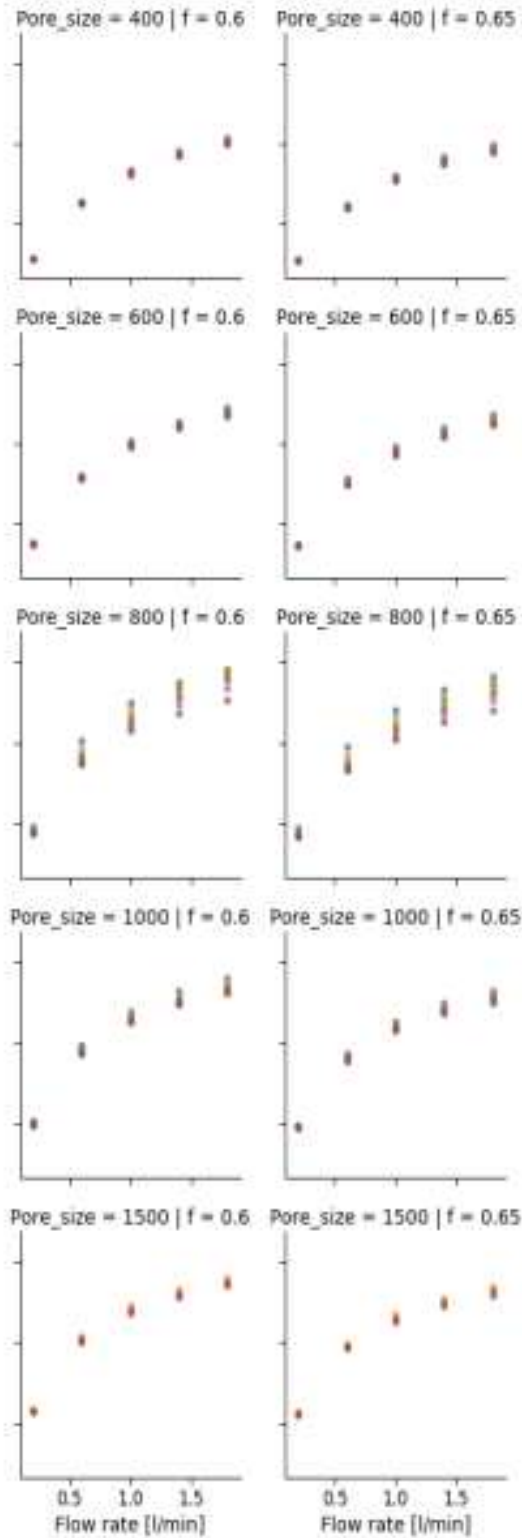

Pore_size $=600 \mid f=0.65$

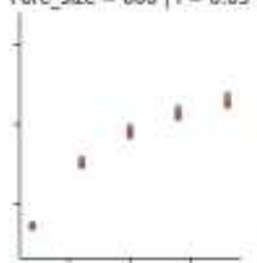

Pore_size $=800 \mid t=0.65$
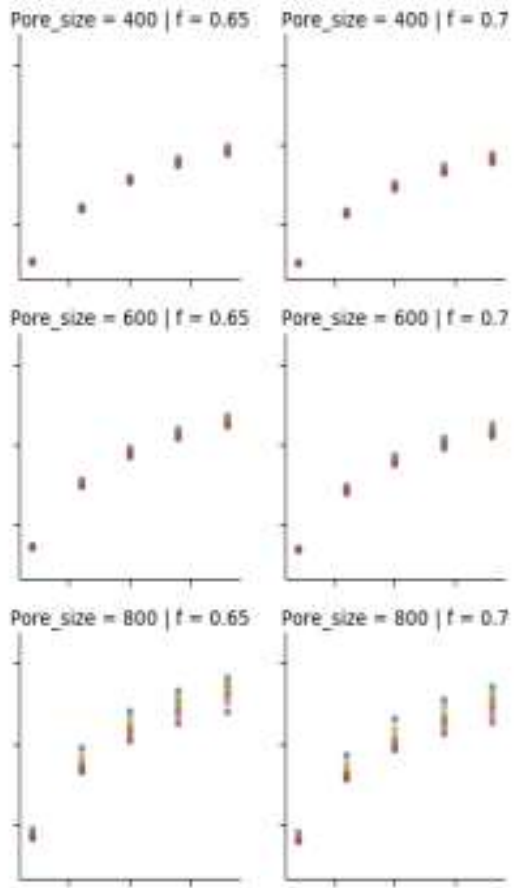

Pore size $=6001 \mathrm{f}=0.7$
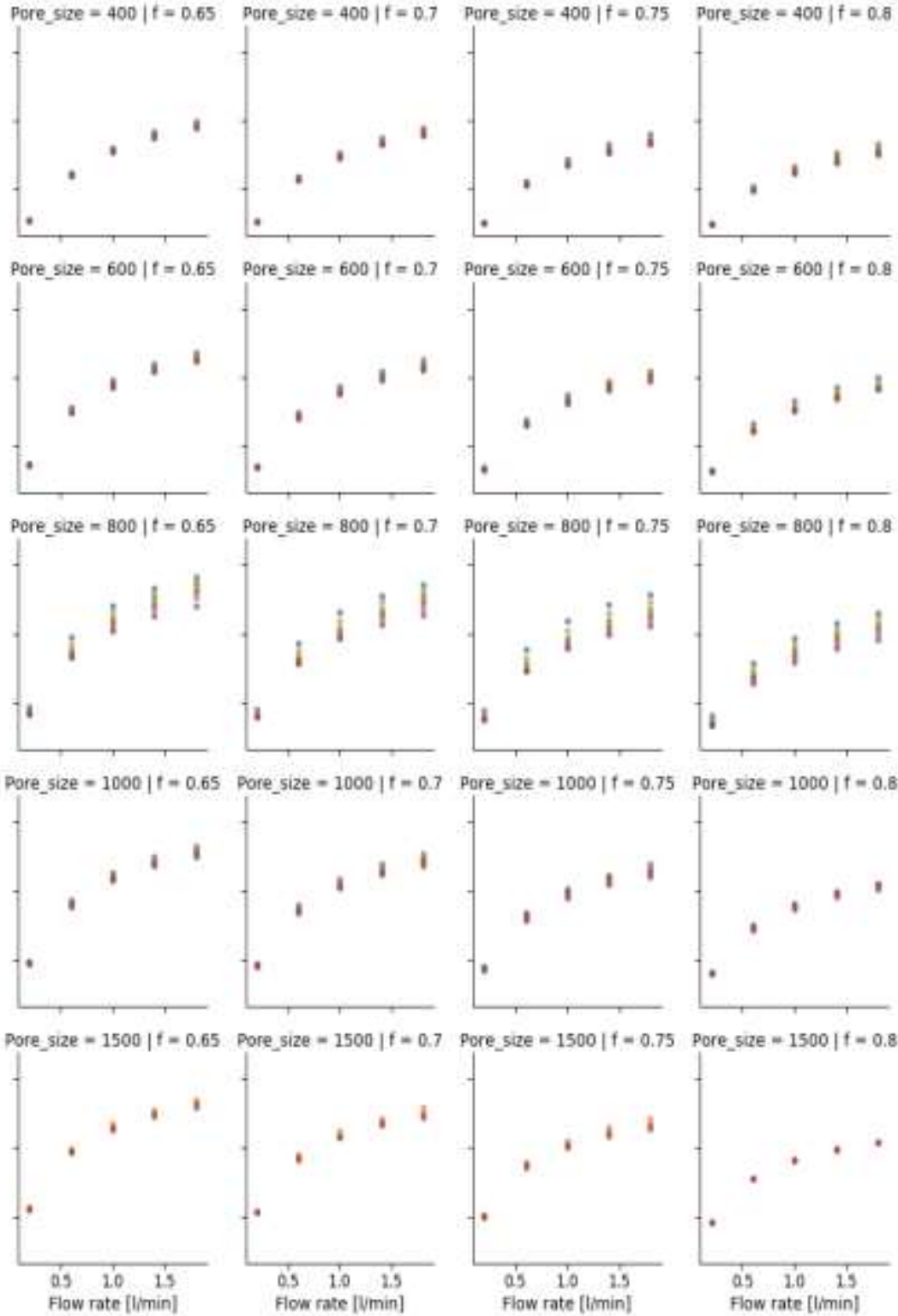

Fig. 3: Heat transfer coefficient of 810 numerical simulations using a representative elementary volume of a porous copper sample.

From Fig. 3, it is possible to observe how the heat transfer coefficient enhances with flow rate (on each individual plot) and pore size (from top to bottom of the figure). Yet, in both cases, the increasing trend is parabolic. For instance, when the flow rate was below $1.0 \mathrm{l}$ min, the heat transfer coefficient increased more rapidly. In the literature, several studies on porous media have shown that the heat transfer coefficient increases with increasing flow rate [23][24]. It is acknowledged that turbulent flow occurs within the porous network with higher flow rates. This turbulence will enhance mixing and provide access to smaller pore connections within the porous metal [25]. This mixing behaviour will increase the heat transfer to the water by forced convection. This can be observed on the samples with a flow rate of $1.81 \mathrm{l} / \mathrm{min}$ and pore size of $800 \mu \mathrm{m}$. These samples exhibited the highest heat transfer coefficient of all. Zhang et al. [26] reported that the best heat transfer performance for the lost carbonate sintering (LCS) porous structures was achieved with a pore size in the range of 425-710 
$\mu \mathrm{m}$. It was also reported that larger and smaller pore sizes underperformed. Small pores lead to higher fluid resistance, and for the larger pores, this means less surface area.

The heat transfer coefficient decreases with porosity (from left to right of the figure). It is shown that the trend was almost linear at first. Later, the heat transfer coefficient decreases more rapidly as the porosity increases. The reason for this is that less surface area will lead to less heat being transferred to the solid walls from the heated source (less conduction of energy) and consequently, less heat being removed from the porous metal through convection. Similar conclusion regarding the effect of porosity on the heat transfer coefficient can be found in the literature for metal foams [27] and also porous metals [28].

In addition, the dataset showed that the heat transfer coefficient decreases slightly with metal particle size. The difference on the heat transfer coefficient due to the range of metal particle sizes studied is it is only observable on the structures with pore size of $800 \mu \mathrm{m}$. This is due to the small range for metal particle size on the dataset.
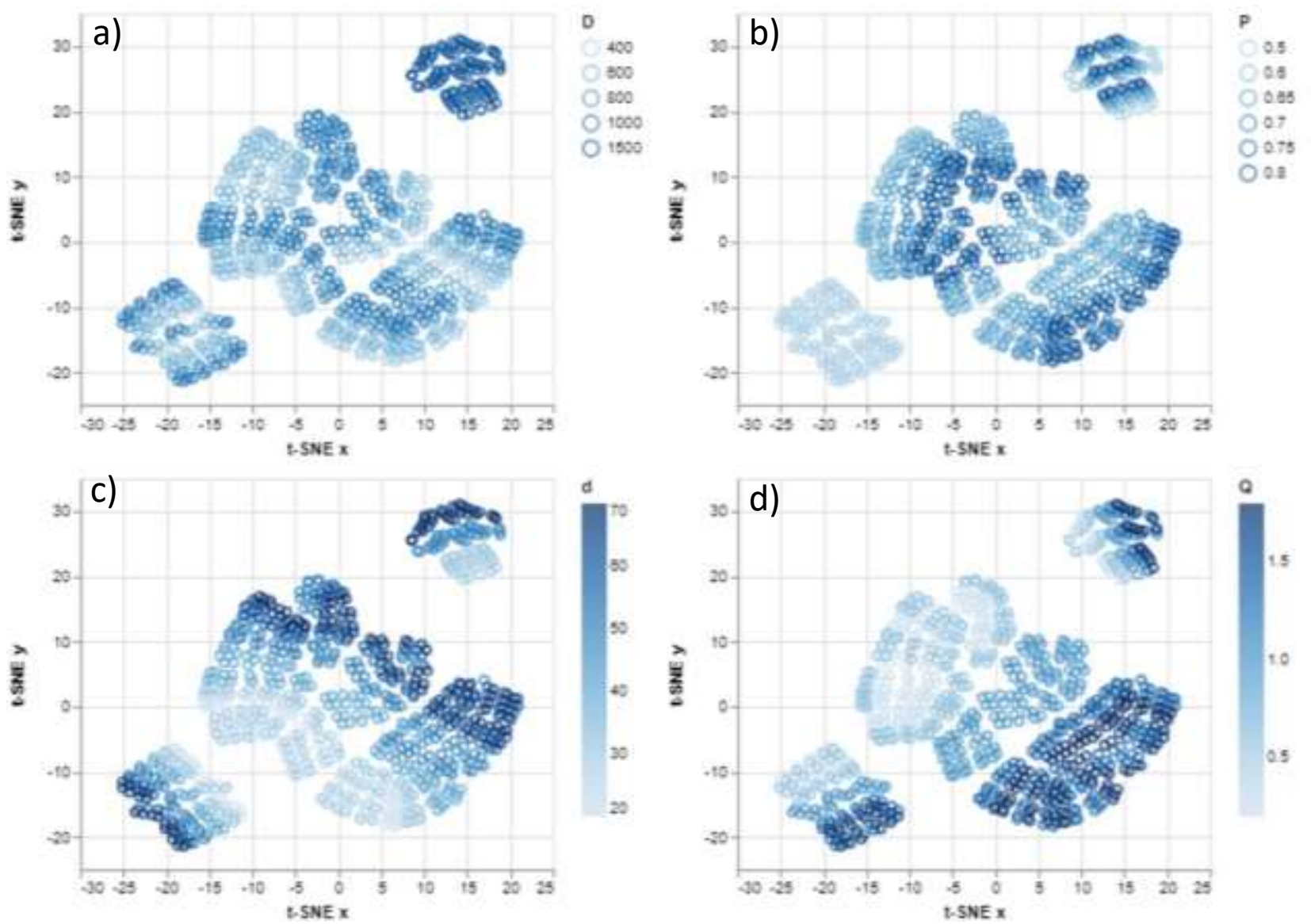

Fig. 4: t-SNE representation of the four parameters chosen for the heat transfer prediction, a) Pore size, b) Porosity, c) Metal particle size, and d) Flow rate.

A t-distributed stochastic neighbour embedding (t-SNE) [29] model was used in order to have a better visualization of the behaviour shown by the input parameters as main descriptors of the heat transfer problem. From Fig. 4 it is possible to observe the existence of three different clustering arrangements. For instance, in Fig. 4 (a), the main cluster corresponds to those samples with pore sizes smaller than $800 \mu \mathrm{m}$, the other 2 clusters are formed by samples with pore size of 800 to 1000 $\mu \mathrm{m}$ (left) and samples with $1500 \mu \mathrm{m}$ (Right). On each cluster is possible to observe different interaction amongst the parameters. However, Fig. 4(b) clearly shows that porosity on the left cluster corresponds to those with values of $50 \%$ and 
$60 \%$. Moreover, Fig 4 (c,d) shows that Metal particle size and flow rate exhibited an increasing or decreasing trend on each of their clusters. Yet, Fig. 4(c) provides some insight on the influence of the metal particle size on the other properties of the material. Xiao [30] described how larger metal particles with a diameter of $300 \mu \mathrm{m}$, can lead to significant decrement on heat transfer coefficient up to 50\%. Thus, a dataset that includes larger metal particles sizes is desirable for future research. An additional t-SNE analysis was carried out in order to establish which clustering provided the best heat transfer performance. The results can be observed in Fig. 5 and it shows that the two clusters on the sides exhibited the best heat transfer coefficient.

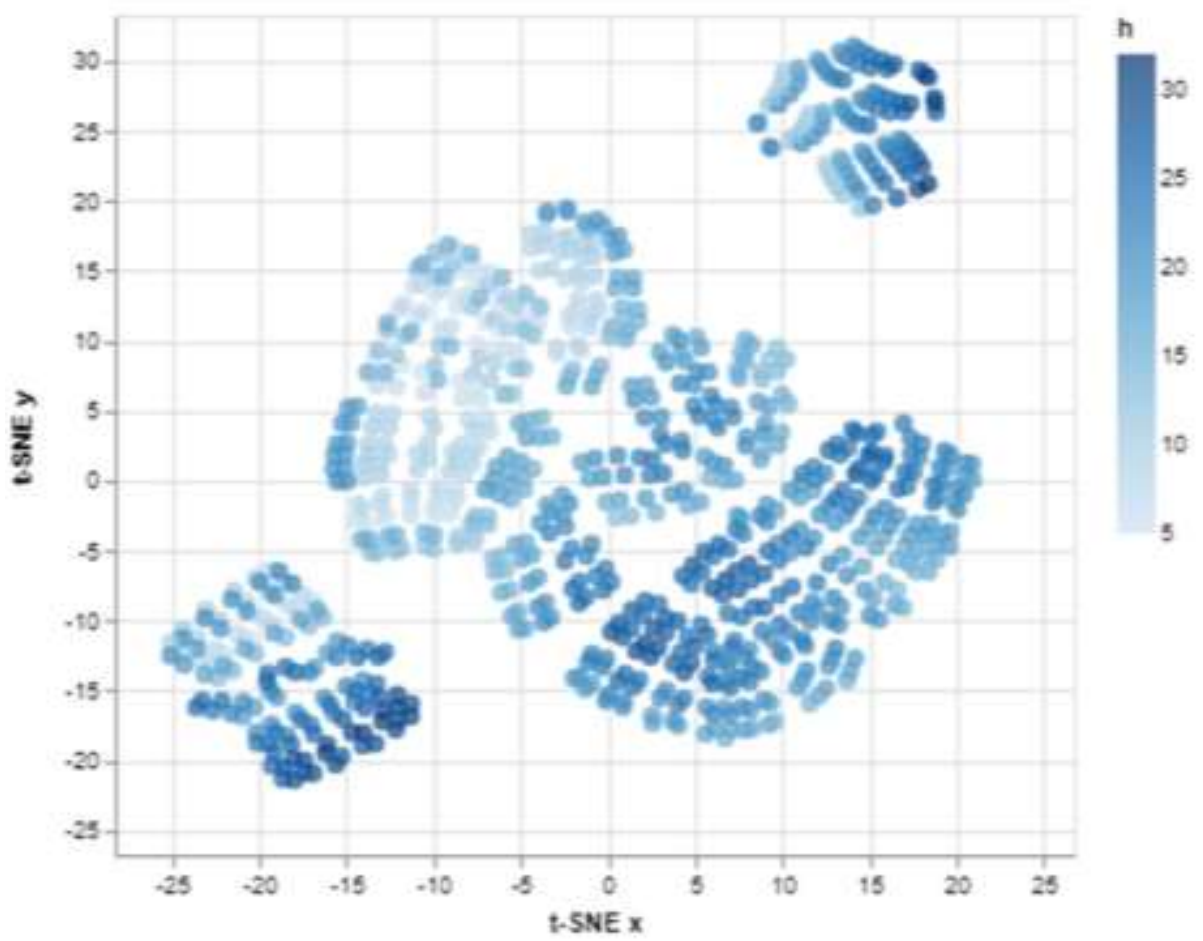

Fig. 5: t-SNE model of the heat transfer coefficient of the porous copper samples.

This model also demonstrates that the highest heat transfer coefficients are obtained on the 2 clusters on the sides. In both cases pore size seems to be an essential factor. Therefore, it is quite important to include pore size on the correlation model for heat transfer calculation. Moreover, only a clear trend of porosity can be observed on the cluster on the left as shown in Fig. 4 (b). This can explain why the combination of properties on the left cluster, seem to have a slightly higher heat transfer coefficient, than the cluster on the right.

To further study the data set of the porous copper, a supervised ML model namely multiple linear regression was performed in order to establish a correlation for the heat transfer coefficient that encompasses different combinations of the input variables. A multiple linear relationship that includes all four parameters (i.e. porosity, pore size, metal particle size and flow rate) was found to have the best fit. The resulting equation is as follows:

$$
h=17.17386+0.006362 D-0.029209 d-18.549759 \varepsilon+9.114513 Q
$$

where $D$ is the the pore diameter given by the $\mathrm{K}_{2} \mathrm{CO}_{3}$ particle size, $d$ is the diameter of the copper particles, $\varepsilon$ is porosity, and $Q$ is the volumetric flow rate. This relationship has a correlation of $86.26 \%$. The correlation value of Eq. (3) is $10 \%$ higher than the correlation obtained when considering only porosity and flow rate. These two parameters have been described in the literature as the main descriptors of the heat transfer analyses [26]. To further analyse the relationship presented in the 
present paper, the predicted values were compared with another relationship found in the literature from Xiao and Zhao [22]. This correlation is presented in Eq. (4) and was obtained by experimental means:

$$
h=226.87(1-\varepsilon) u^{0.60}+5.78 u^{0.15}
$$

where $D$ is the Darcian velocity. The two correlations were compared using the experimental data for heat transfer coefficients reported by Baloyo [28]. The resulting values were plotted against the real values in order to show their accuracy to predict the heat transfer coefficient. Any points lying on the diagonal dashed line will indicate a perfect correlation between the model and the experimental data used as testing data set. The scatter plot is shown in Fig. 66. It can be seen that Eq. (3) provides a better correlation than Eq. (4) for the samples used as testing dataset. The predicted values from Eq. (4) showed to diverge as the calculated value increased. In most of the cases, the difference between the predicted values and the real values were about 50\% larger. In contrast, values predicted using Eq. (3) are within a range of $\pm 15 \%$ different from the experimental results. Additionally, it is possible to observe an increasing trend in the same direction as the experimental values.

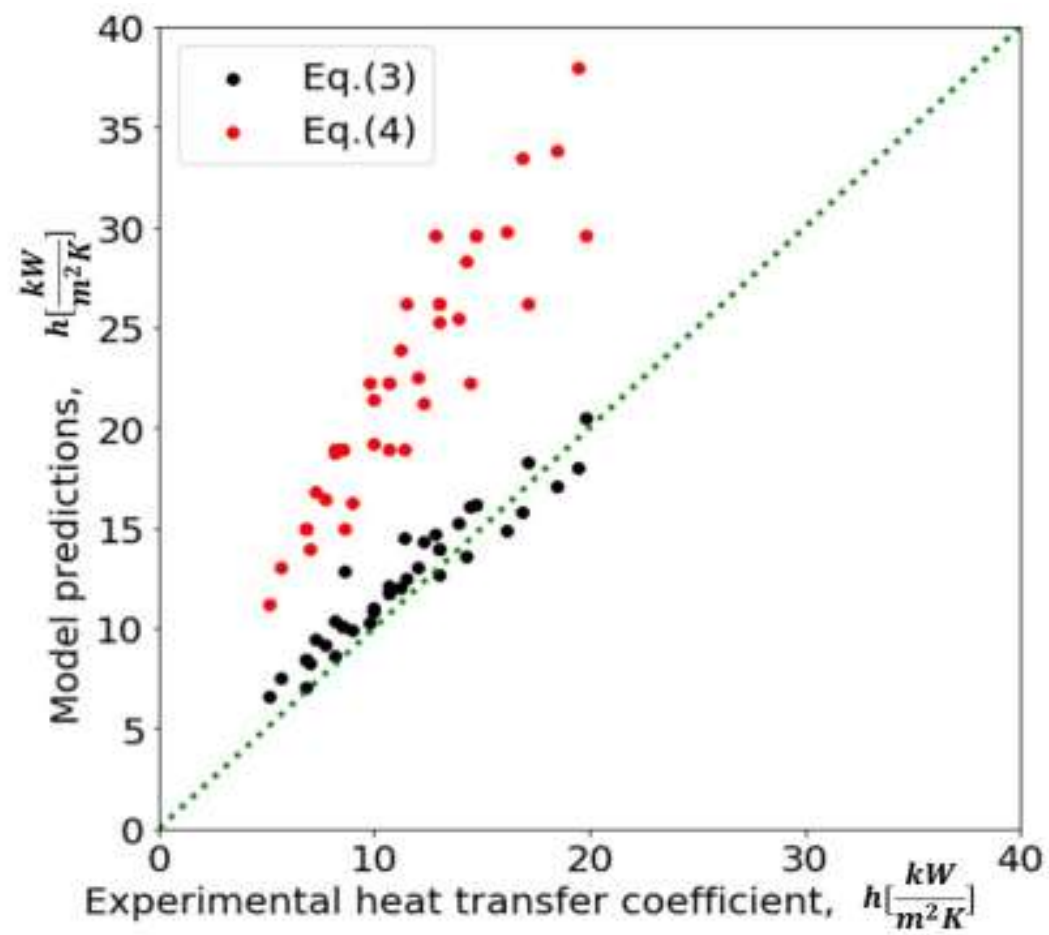

Fig. 6: Performance of the two correlations in estimating the heat transfer coefficient of 40 samples of porous copper.

\section{Conclusions}

The data-driven approach described above has successfully calculated a correlation for the heat transfer coefficient of the porous copper. From the data, it was observed that the heat transfer coefficient decreased linearly with increasing porosity, because higher porosity means less heat being transferred to the metal matrix by conduction. Increasing water flow rate increased the heat transfer performance in all cases. Increasing pore size increased the heat transfer coefficient, especially marked when increasing pore size from $400 \mu \mathrm{m}$ to $600 \mu \mathrm{m}$. Increasing pore size further led to smaller enhancements on the heat transfer coefficient. Metal particle size had a lesser effect on heat transfer coefficient. 
Overall, water flow rate and porosity showed major influences on the heat transfer coefficient. The multiple linear correlation of the heat transfer coefficient with pore size, metal particle size, porosity, Darcian velocity exhibited an adjusted adjusted and more accurate model with a $R^{2}$ value of 0.86 .

\section{References}

[1] B. D. Conduit, N. G. Jones, H. J. Stone, and G. J. Conduit, "Probabilistic design of a molybdenum-base alloy using a neural network," Scr. Mater., vol. 146, pp. 82-86, 2018.

[2] K. Matouš, M. G. D. Geers, V. G. Kouznetsova, and A. Gillman, "A review of predictive nonlinear theories for multiscale modeling of heterogeneous materials," J. Comput. Phys., vol. 330, pp. 192-220, 2017.

[3] T. Lookman, P. V. Balachandran, D. Xue, J. Hogden, and J. Theiler, "Statistical inference and adaptive design for materials discovery," Curr. Opin. Solid State Mater. Sci., vol. 21, no. 3, pp. 121-128, 2017.

[4] J. H. Panchal, S. R. Kalidindi, and D. L. McDowell, "Key computational modeling issues in Integrated Computational Materials Engineering," CAD Comput. Aided Des., vol. 45, no. 1, pp. 4-25, 2013.

[5] A. Gupta, A. Cecen, S. Goyal, A. K. Singh, and S. R. Kalidindi, "Structure-property linkages using a data science approach: Application to a non-metallic inclusion/steel composite system," Acta Mater., vol. 91, pp. 239-254, 2015.

[6] K. Rajan, "Materials informatics," Mater. today, pp. 38-45, 2005.

[7] K. Takahashi and Y. Tanaka, "Material synthesis and design from first principle calculations and machine learning," Comput. Mater. Sci., vol. 112, pp. 364-367, 2016.

[8] A. Smola and S. V. N. Vishwanathan, Introduction to Machine Learning, 1st Ed. Cambridge, UK: Cambridge University Press, 2008.

[9] D. H. Wolpert and W. G. Macready, "Wolpert -- No Free Lunch Theorems.pdf," Trans. Evol. Comput., vol. 1, no. 1, pp. 67-82, 1997.

[10] S. Ramakrishna et al., "Materials informatics," J. Intell. Manuf., vol. 58, no. 6, pp. 1-20, 2018.

[11] L. Ward and C. Wolverton, "Atomistic calculations and materials informatics: A review," Curr. Opin. Solid State Mater. Sci., vol. 21, no. 3, pp. 167-176, 2017.

[12] W. Lu, R. Xiao, J. Yang, H. Li, and W. Zhang, "Data mining-aided materials discovery and optimization," J. Mater., vol. 3, no. 3, pp. 191-201, 2017.

[13] E. Bélisle, Z. Huang, S. Le Digabel, and A. E. Gheribi, "Evaluation of machine learning interpolation techniques for prediction of physical properties," Comput. Mater. Sci., vol. 98, pp. 170-177, 2015.

[14] B. D. Conduit, N. G. Jones, H. J. Stone, and G. J. Conduit, "Design of a nickel-base superalloy using a neural network," Mater. Des., vol. 131, no. April, pp. 358-365, 2017.

[15] N. Artrith and A. Urban, "An implementation of artificial neural-network potentials for atomistic materials simulations: Performance for TiO2," Comput. Mater. Sci., vol. 114, pp. 135-150, 2016.

[16] E. Avalos Gauna and Y. Zhao, "Numerical Simulation of Heat Transfer in Porous Metals for Cooling Applications," Metall. Mater. Trans. B, 2017.

[17] C. Y. Zhao, T. J. Lu, H. P. Hodson, and J. D. Jackson, "The Temperature Dependence of Effective Thermal Conductivity of Open-Celled Steel Alloy Foams," Mater. Sci. Eng., vol. 367, pp. 123-131, 2004.

[18] H. Ohno, "Uniforming the dimensionality of data with neural networks for materials informatics," Appl. Soft Comput. J., vol. 46, pp. 17-25, 2016.

[19] Edgar Avalos-Gauna, "Numerical Simulation of Active Cooling Using Porous Metals," University of Liverpool, 2017.

[20] N. Dukhan, Ö. Bağci, and M. Özdemir, "Experimental Flow in Various Porous Media and Reconciliation of Forchheimer and Ergun Relations," Exp. Therm. Fluid Sci., vol. 57, pp. 425-433, 2014.

[21] T. J. Lu, H. A. Stone, and M. F. Ashby, "Heat Transfer in Open-Cell Metal Foams," Acta Mater., vol. 46, no. 10, pp. 3619-3635, 1998.

[22] Z. Xiao and Y. Zhao, "Heat Transfer Coefficient of Porous Copper with Homogeneous and Hybrid Structures in Active Cooling,” J. Mater. Res., vol. 28, no. 17, pp. 2545-2553, 2013. 
[23] A. Kopanidis, A. Theodorakakos, E. Gavaises, and D. Bouris, "3D Numerical Simulation of Flow and Conjugate Heat Transfer Through a Pore Scale Model of High Porosity Open Cell Metal Foam," Int. J. Heat Mass Transf., vol. 53, no. 11-12, pp. 2539-2550, 2010.

[24] M. Bai and J. N. Chung, "Analytical and Numerical Prediction of Heat Transfer and Pressure Drop in Open-Cell Metal Foams," Int. J. Therm. Sci., vol. 50, no. 6, pp. 869-880, 2011.

[25] E. Avalos Gauna and Y. Zhao, "Numerical Simulation of Heat Transfer in Porous Metals for Cooling Applications," in SESSION 2: SOLIDIFICATION AND CASTING, SALON B, 2016, no. October, p. 2016.

[26] L. Zhang, D. Mullen, K. Lynn, and Y. Zhao, "Heat Transfer Performance of Porous Copper Fabricated by the Lost Carbonate Sintering Process," in Mater. Res. Soc. Symp. Proc, 2009, vol. 1188.

[27] S. Mahjoob and K. Vafai, "A Synthesis of Fluid and Thermal Transport Models for Metal Foam Heat Exchangers," Int. J. Heat Mass Transf., vol. 51, no. 15-16, pp. 3701-3711, 2008.

[28] J. M. Baloyo, "Porous Metals with Novel Structures for Optimum Heat Exchange Performance," PhD Thesis, University of Liverpool, 2016.

[29] L. Van der Maaten, "Barnes-Hut-SNE," Pattern recognition and Bioinformatics, pp. 1-11, 2013.

[30] Z. Xiao, "Heat Transfer, Fluid Transport and Mechanical Properties of Porous Copper Manufactured by Lost Carbonate Sintering," PhD Thesis, 2013. 